\title{
"Theoretical and experimental analysis of an innovative dual-axis tracking linear Fresnel lenses concentrated solar thermal collector"
}

\author{
Simoni Perini: Lark Energy, Larkfleet House, Falcon Way, Bourne, Lincolnshire, PE10 0FF \\ Xavier Tonnellier: Cranfield University, Bedford, Bedfordshire MK43 0AL \\ Peter King: Cranfield University, Bedford, Bedfordshire MK43 0AL \\ Christopher Sansom: Cranfield University, Bedford, Bedfordshire MK43 0AL \\ Corresponding author: Christopher Sansom: c.l.sansom@cranfield.ac.uk
}

\begin{abstract}
Linear concentrating solar thermal systems offer a promising method for harvesting solar energy. In this paper, a model for a novel linear Fresnel lens collector with dual-axis tracking capability is presented. The main objective is to determine the performance curve of this technology by means of both experiment and theoretical analysis. A mathematical model including the optical model of the concentrator and the heat transfer model of the receiver pipe was developed. This tool was validated with experimental data collected using a proof of concept prototype installed in Bourne, UK. The performance curve of the collector was derived for temperatures between $40^{\circ} \mathrm{C}$ and $90^{\circ} \mathrm{C}$. The results show that the global efficiency of the collector is limited to less than $20 \%$. The energy losses have been analysed. The optical losses in the lens system accounts for $47 \%$ of the total energy dissipated. These are due to absorption, reflection and diffraction in the Fresnel lenses. Furthermore manufacturing error in the lens fabrication has to be considered. One third of the solar radiation collected is lost due to the low solar absorptance of the receiver pipe. Thermal radiation and convection accounts for $6 \%$ of the total as relatively low temperatures (up to $90{ }^{\circ} \mathrm{C}$ ) are involved. In order to increase the performance of the system, it is recommended to install an evacuated receiver and to insulate the recirculation system. Considering data from manufacturers, these improvements could increase the global efficiency up to $55 \%$. Utilising the results from this work, there is the intention of building an improved version of this prototype and to conduct further tests.
\end{abstract}

Keywords: Solar Energy, Fresnel lens, Two-axis tracking system

\section{Highlights:}

- The optical and thermal models of a novel linear Fresnel lens collector with 2D tracking are presented and validated using experimental data.

- The performance curve shows that the global efficiency of the collector is limited to $20 \%$.

- Optical losses in the lens system accounts for $47 \%$ of the total energy dissipated. $33 \%$ of the solar radiation is lost due to low solar absorptance of the receiver pipe.

- Installing an evacuated receiver and insulating the recirculation system could increase the global efficiency up to $55 \%$.

\section{Table of Contents}

1 Introduction:

1.1 Solar Thermal energy potential and linear concentrated collector systems ............................ 3

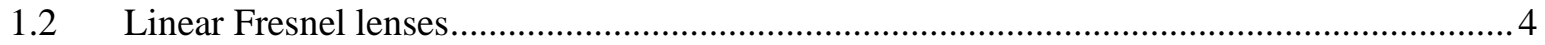

1.3 Linear Fresnel lenses for thermal applications................................................................. 


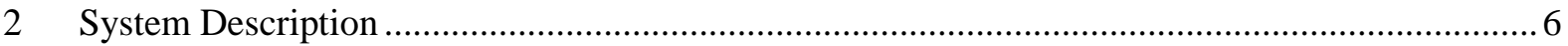

2.1 Dual axis linear Fresnel concentrator experimental prototype............................................ 6

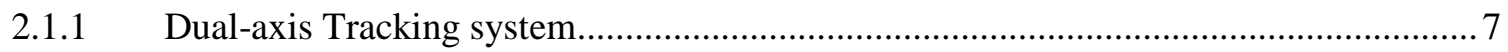

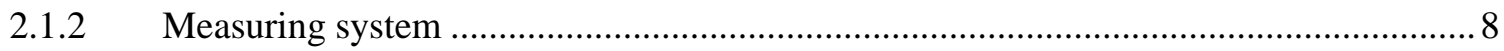

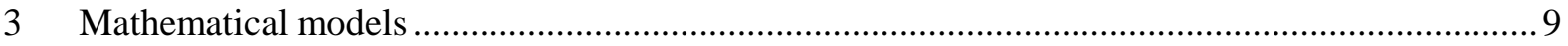

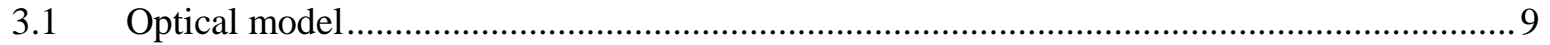

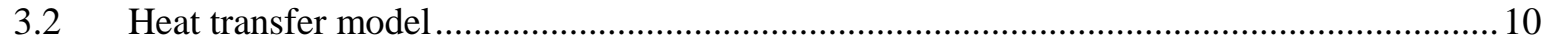

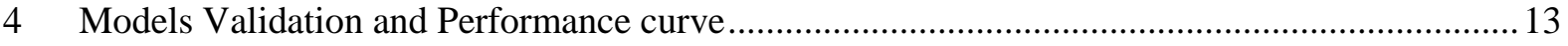

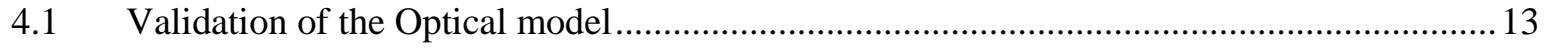

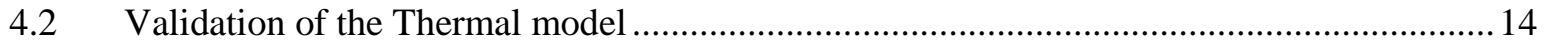

$4.3 \quad$ Performance Curve and losses analysis ......................................................................... 17

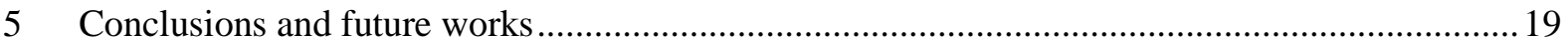

\section{List of Figures}

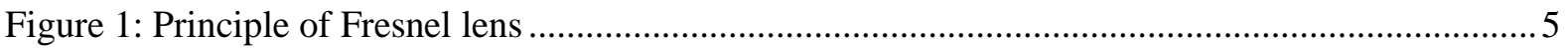

Figure 2: Experimental prototype scheme installed in Bourne, UK................................................. 6

Figure 3: Description of the three section of the optical system. The yellow line represent the axis of

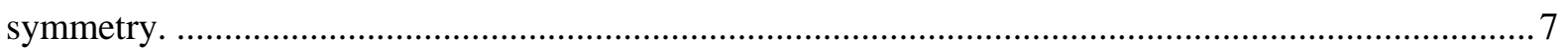

Figure 4: Schematic diagram and measuring system of the dual-axis linear Fresnel lens collector ....... 8

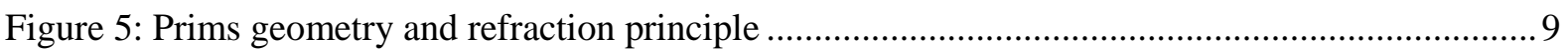

Figure 6: Thermal resistance network for the absorber cross section ............................................ 10

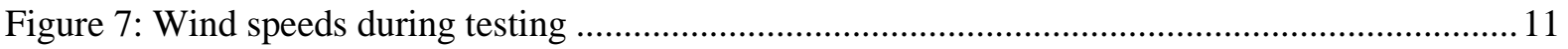

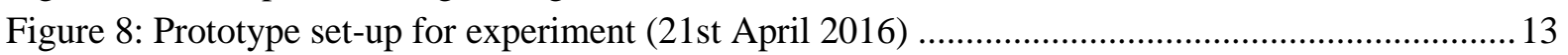

Figure 9: a) Concentrated beam during experiment (21st April 2016) b) Flux distribution according to

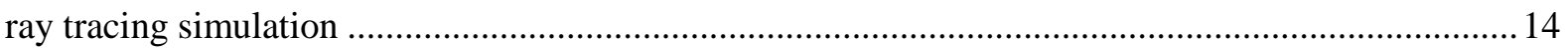

Figure 10: Measured and calculated temperature profiles for the $4^{\text {th }}$ of June $2015 \ldots \ldots \ldots \ldots \ldots \ldots \ldots \ldots \ldots \ldots . . . . . . . . . . . .15$

Figure 11: Measured and calculated temperature profiles for the $11^{\text {th }}$ of June 2015 .......................... 15

Figure 12: Comparison of experimental and simulation results for the 11th of June 2015 ................... 16

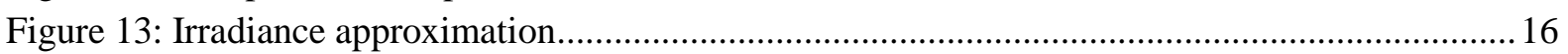

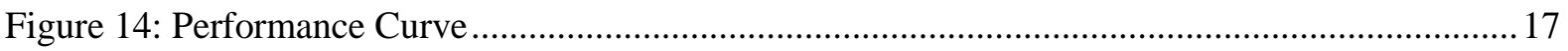

Figure 15: Thermal losses breakdown for the experiment on the 4th June 2015 ............................... 18

Figure 16: Thermal losses analysis for the experiment on the 4th June 2015 ................................... 18

\section{List of Tables}

Table 1: List of heat transfer coefficient used in the model.

\section{Nomenclature}

A Aperture area $\left[\mathrm{m}^{2}\right]$

$\mathrm{C}_{\mathrm{p}} \quad$ Heat capacity at constant pressure $[\mathrm{kJ} / \mathrm{kg} \mathrm{K}]$

D Diameter $[\mathrm{m}]$

$\mathrm{f} \quad$ Focal distance $[\mathrm{m}]$ 


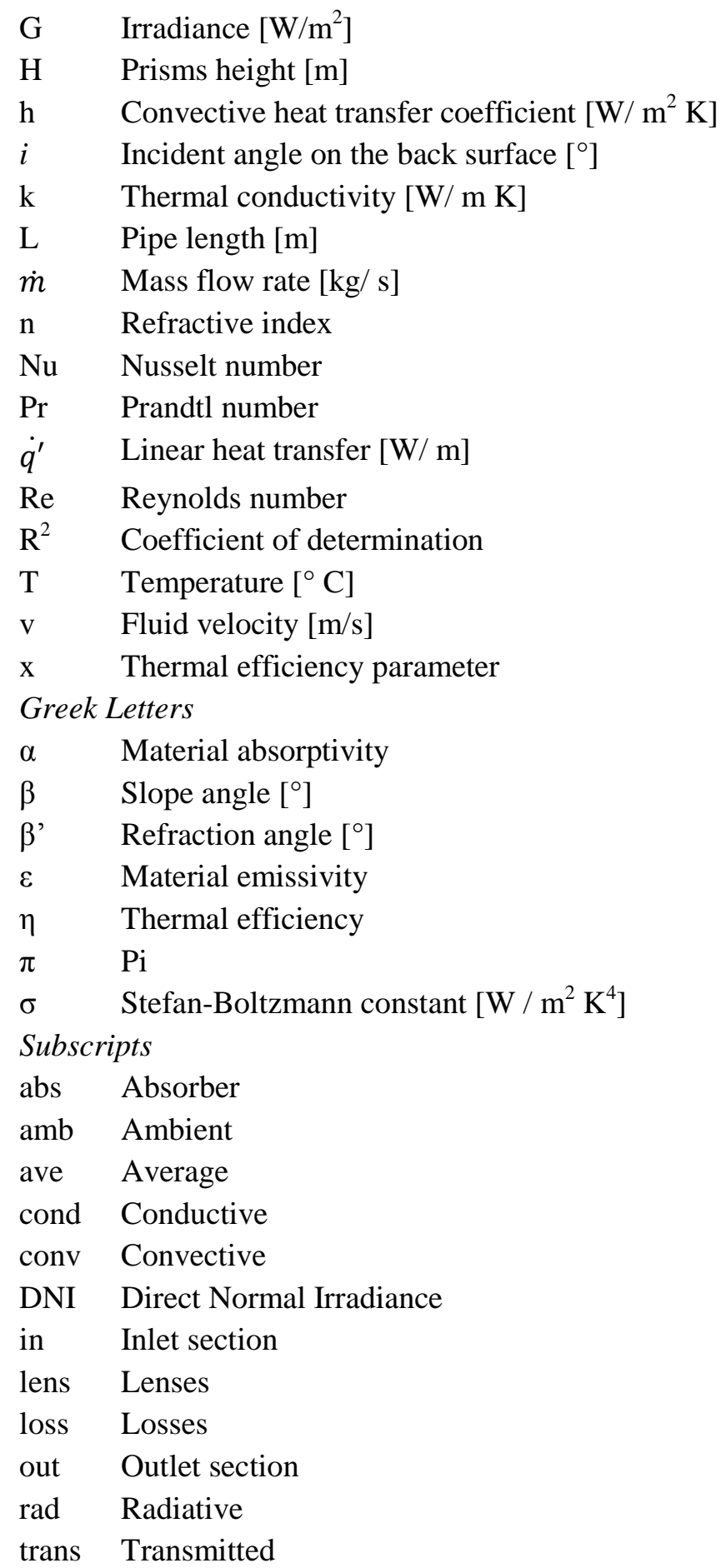

\section{Introduction:}

\subsection{Solar Thermal energy potential and linear concentrated collector systems}

Heat represents $76 \%$ of the global industrial energy consumption and $57 \%$ of this demand is required at temperatures below $400^{\circ} \mathrm{C}$ (Rawlins and Ashcroft, 2013). Solar thermal energy can be an extremely convenient and sustainable source of heat, but only concentrated system can reach temperature levels up to $250{ }^{\circ} \mathrm{C}$, as required in most industrial processes. In several industry sectors, such as food, textiles and chemicals the share of heat demand required at temperatures below $250^{\circ} \mathrm{C}$ is around $60 \%$. 
The United Nations Industrial Development Organization (UNIDO) estimated the potential of concentrated solar technologies in industry to be 500 million GJ in 2050 (Taibi et al., 2010).

Flat plate solar collectors are designed to deliver lower temperature levels (up to $150^{\circ} \mathrm{C}$ ) (IRENA, 2015). Concentrated solar collectors can produce temperatures up to $3000^{\circ} \mathrm{C}$ in central receiver concentrated solar power (CSP) plants. Concentrated systems (linear or point focus) have higher efficiency at high temperatures because the ratio of thermal energy loss to total incident solar radiation on the receiver decreases significantly (Zhai et al., 2010). Current medium temperature collectors that can meet the industrial process heating requirement are based on concentrated technologies developed for power generation purposes. Parabolic trough collectors are based on thick (3-4mm) glass second surface mirrors which have high cost and are difficult to integrate with existing industrial processes (Kumar et al., 2015). Therefore, there is a need for the development of innovative concentrated solar thermal collectors for applications in industrial processes.

Linear concentrating solar thermal systems offer a promising method for harvesting solar energy (Zhai et al., 2010). There are three main linear concentrator technologies: parabolic trough solar concentrators, linear reflected Fresnel concentrators and linear refracted Fresnel lens arrangements. Most published studies on linear concentrating collectors are related to parabolic trough systems with evacuated tubular absorbers, illustrating the emphasis on power generating applications (FernándezGarcía et al., 2010; Xie et al., 2011). PMMA Fresnel lenses have the disadvantage of transmittance loss through the lens, but also have advantages in comparison with small-scale reflecting concentrators, which tend to mitigate this radiation disadvantage. These include (Kumar et al., 2015):

- Ultra high temperatures can be generated with Fresnel CSP technology, which is suitable for various thermal energy applications.

- A compact and maintenance free concentrator.

- $\quad$ Fresnel-CSP based Direct Steam Generation (DSG) systems do not need a heat transfer fluid or heat exchanger.

- $\quad$ A high thermal efficiency, compact plant size, low cost, and a shorter payback period.

- $30 \%$ of industrial energy consumption required as process heat is in the low to medium temperature range i.e. $80^{\circ} \mathrm{C}-250^{\circ} \mathrm{C}$, and PMMA Fresnel lens technology is ideally suited to this range.

\subsection{Linear Fresnel lenses}

The flat linear Fresnel lens is considered to be a suitable concentrator for both photo-thermal and photovoltaic conversion of solar radiation (Al-Jumaily and Al-Kaysi., 1998; Boyd et al., 1976; Franc et al., 1986). A Fresnel lens is an optical component which can be used as a lightweight alternative to conventional continuous surface optics. Fresnel lenses also possess the advantage of higher flexibility in optical design when compared to conventional mirror concentrators (Leutz and Suzuki, 2001). The refractive capacity of standard lenses depends mainly on the curvature of the surface. Removing as much of the optical material as possible while still maintaining the curvature will create the same effect. This is obtained by means of a series of parallel grooves with different angles. The solar radiation passing through each groove is refracted at a slightly different angle and converges on the focal line of the lens (Figure 1) (Davis and Kuhnlenz, 2007). 


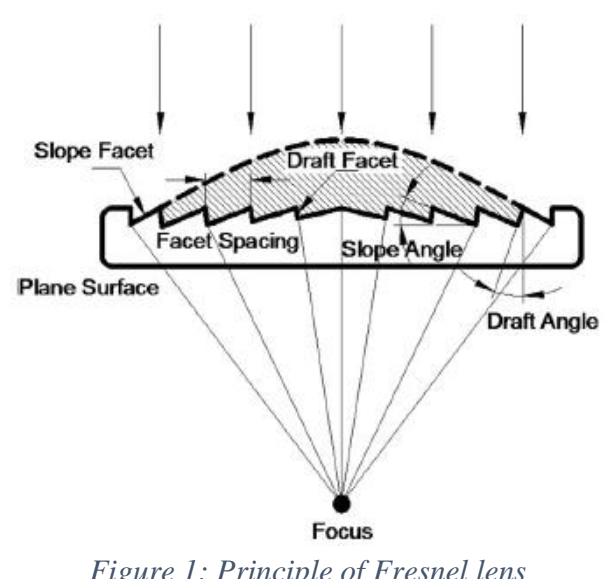

\subsection{Linear Fresnel lenses for thermal applications}

Research into Fresnel lenses is mainly focused on point focus concentrating photovoltaic systems and there are limited studies available about Fresnel linear concentrating solar collectors for hybrid photovoltaic and thermal systems.

A first theoretical performance analysis of a linear Fresnel concentrating collector with cylindrical blackbody absorber was carried out in 1976. The study demonstrated that the concept output temperatures were between $130^{\circ} \mathrm{C}$ and $370^{\circ} \mathrm{C}$ (Boyd et al., 1976). Franc et al. (1986) studied and tested a stationary linear Fresnel lens system with movable absorber. The experiments showed a yearly gain of $360 \mathrm{kWh} / \mathrm{m}^{2}$ under climatic condition in Central Europe. AL-Jumaily and Al-Kaysi (1998) studied a solar collector which consisted of two flat linear Fresnel lenses made of glass. The system was designed to track the sun in two directions. The peak measured efficiency was $58 \%$ in Iraq. The two lens system reached an outlet temperature of $37^{\circ} \mathrm{C}$.

Zhai et al. (2010) conducted a theoretical and experimental analysis on a flat linear Fresnel lens being integrated with an evacuated tube receiver. It was reported that the performance of the Fresnel lens collector was higher compared to a simple non-concentrating collector and that the system efficiency can reach as high as 55\% at working temperatures of $90{ }^{\circ} \mathrm{C}$. Zheng et al. (2014) designed a cylindrical compound Fresnel lens system and they produced a small scale prototype to measure the optical efficiency. An optical efficiency of up to $84 \%$ was measured. The collector was able to deliver heat at the average outlet temperature of $27^{\circ} \mathrm{C}$. In 2011, the European project Efisol demonstrated the integration of a Fresnel concentrator with an Organic Rankine Cycle engine and thermal storage [Linaric, 2013]. This fully functional prototype was developed and achieved temperatures up to 150 ${ }^{\circ} \mathrm{C}$.

More recently, researchers have focused on the analysis of the receiver for linear Fresnel lens systems. Lin et al. (2014) investigated the performance of a linear Fresnel lens solar collector using different cavity receivers. They found that the receiver design with a triangular cavity reached the highest efficiency. More recently, Manikumar et al. (2014) performed a numerical simulation on a trapezoidal cavity receiver; aiming for a better design with maximum heat transfer rate as well as optimal outlet temperature. The overall heat loss coefficient simulated was in the range of $8-11 \mathrm{~W} \mathrm{~m}^{-2} \mathrm{~K}^{-1}$ at operating temperatures of $130-180^{\circ} \mathrm{C}$.

\subsection{Objectives:}

From the current literature review, limited published works were found related to the performance of linear Fresnel lenses made of plastic materials with a dual-axis tracking system for thermal applications. In this study, a model for a dual-axis tracking flat acrylic linear Fresnel lens concentrator 
is developed and validated. The main objective of this work is to determine the performance curve of this innovative collector by means of both experiment and theoretical analysis.

\section{System Description}

\subsection{Dual axis linear Fresnel concentrator experimental prototype}

The experimental prototype used in this study consists of an array of acrylic (PMMA) linear Fresnel lenses which focuses the solar radiation onto a receiver pipe (Figure 2).

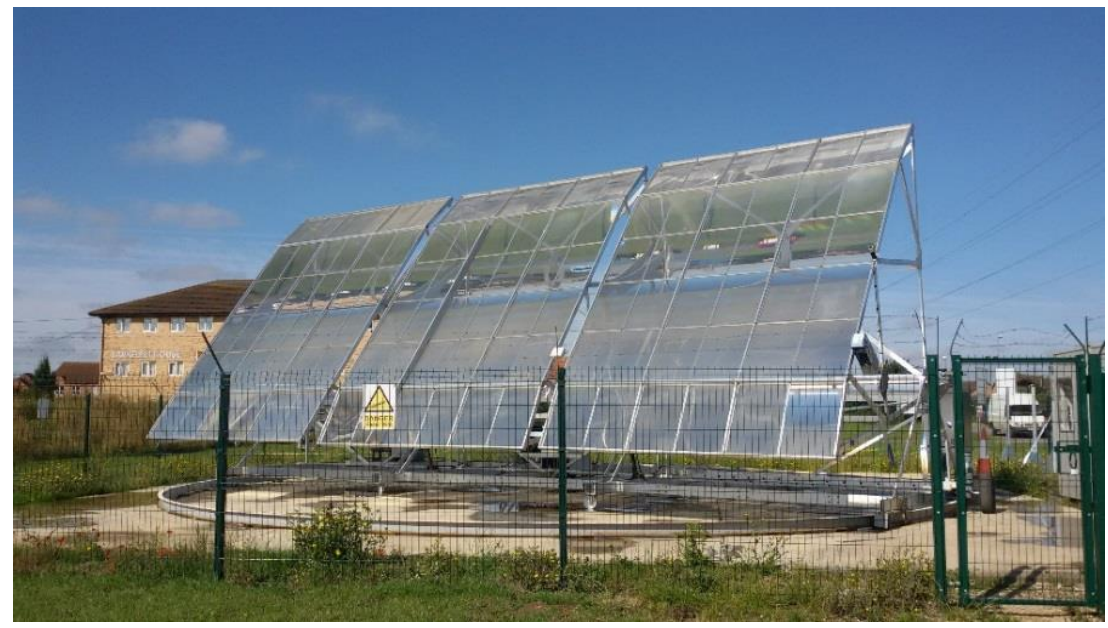

Figure 2: Experimental prototype scheme installed in Bourne, UK.

The prototype consist of three modules of 36 Fresnel lenses having a total aperture area of $75 \mathrm{~m}^{2}$. The aluminium structure hosting the lenses is maintained normal to the sun rays throughout the day using a $2 \mathrm{D}$ tracking system. Hydraulic pistons are responsible for the vertical movement of the frame. An electric slew motor provides the torque to rotate the system around the central pivoting point. An automatic dual axis tracking system controls the axis motions and ensures alignment with the sun. The working fluid is circulated in a copper piping system using two circulation pumps. Water is used in the current version of the prototype. A buffer tank is installed close to the outlet section of the receiver. The tank is not insulated. Excess heat is dissipated through a fanned air heat exchanger to the surroundings.

The system operates in recirculation mode: the water is heated in the absorber exposed to the concentrated solar radiation and it is recirculated back to the inlet section. When the temperature at the outlet section of the absorber approaches $100{ }^{\circ} \mathrm{C}$, the water can be diverted to the heat exchanger for cooling. The current setting of the prototype does not allow the generation of steam for safety reasons.

The receiver is a 13 meters carbon steel pipe (schedule 40). Each Fresnel lens consists of a panel of 1 $\mathrm{m}$ in length and $0.7 \mathrm{~m}$ in width with grooves facing inwards. The focal length of each lens (Lens 1 , Lens 2, and Lens 3) is $2.6 \mathrm{~m}$, and the prism size is 0.5 millimetres. The lenses were fabricated using acrylic resin (polymethylmethacrylate, PMMA) through moulding. They have been manufactured in three symmetrical sections. Lenses 1 and 2 are aligned on the same plane, while lens 3 is tilted by 10 degrees to ensure that the concentrated solar radiation is focused on the receiver tube. The current work considers a sub-section of the total experimental rig that encompasses only lenses 1 and 2 (Figure 3). Lens 3 has not been included in the modelling or experimental work because optical analysis and tests have proven that the Lens 3 section has been manufactured incorrectly. Tests show that Lens 3 section is not focusing energy on the target (the receiver). 


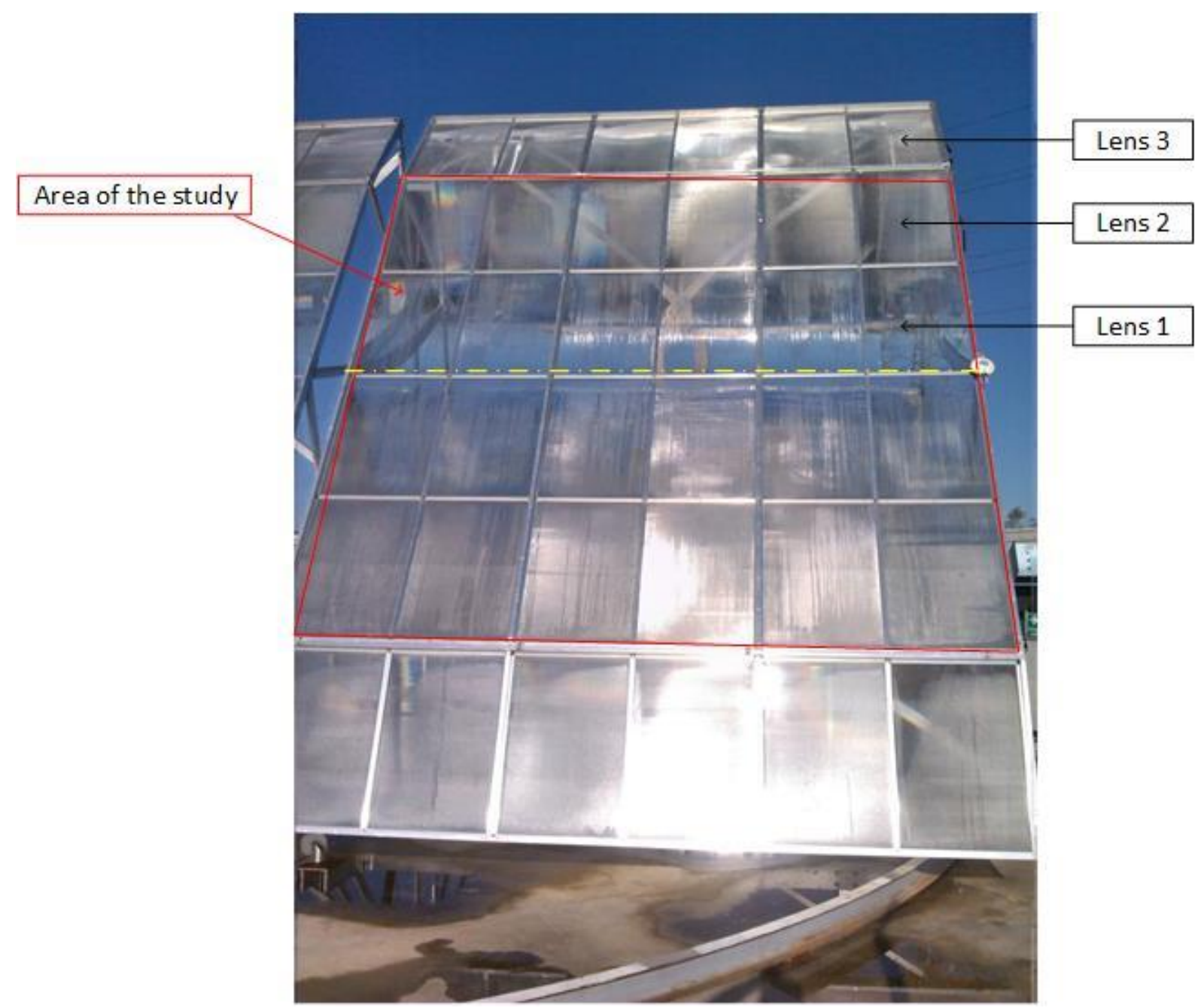

Figure 3: Description of the three section of the optical system. The yellow line represent the axis of symmetry.

\subsubsection{Dual-axis Tracking system}

Solar tracking methods can be divided into two broad categories, namely passive tracking and active tracking. The active electronic systems generally exhibit improved reliability and tracking accuracy. These can be further subdivided into open-loop, closed-loop and mixed control system. In this study, a closed-loop control method is used. The control unit (Horner XLT with special Firmware) compares the calculated position of the sun with the actual position of the prototype. The sun position is obtained using known sun algorithms based on site location and solar time (Reda et al., 2008). The geographical position of the rig is acquired from a GPS system installed on site. The actual position of the prototype is determined by the elevation angle and horizontal angle (angular distance from geographical North). The elevation angle is measured using an inclinometer with a 0.1 degree resolution attached to the tilting frame supporting the lenses. The horizontal angle (azimuth) is measured using an encoder installed on the pivoting mast of the prototype. When the difference between the tilting angle and the sun elevation is larger than 0.5 degree, the control unit moves the prototype to re-focus the lenses. The accuracy for the azimuth angle is 1 degree. The motor is controlled by an inverter drive while the control unit is connected to forward/ reverse digital outputs of the inverter. The pistons are controlled by digital outputs which operate the pump solenoids via relays, whilst a second set of relays simultaneously start the hydraulic pump. The control unit 
compares the measured position and the sun angles every 20 milliseconds. The control unit is supplied by a single phase 230 Volt input. The hydraulic system is supplied by 12 Volt direct current. The electric motor needs a three phase 230 Volt input.

\subsubsection{Measuring system}

The measuring system installed on the experimental prototype consists of the following equipment (Figure 4):

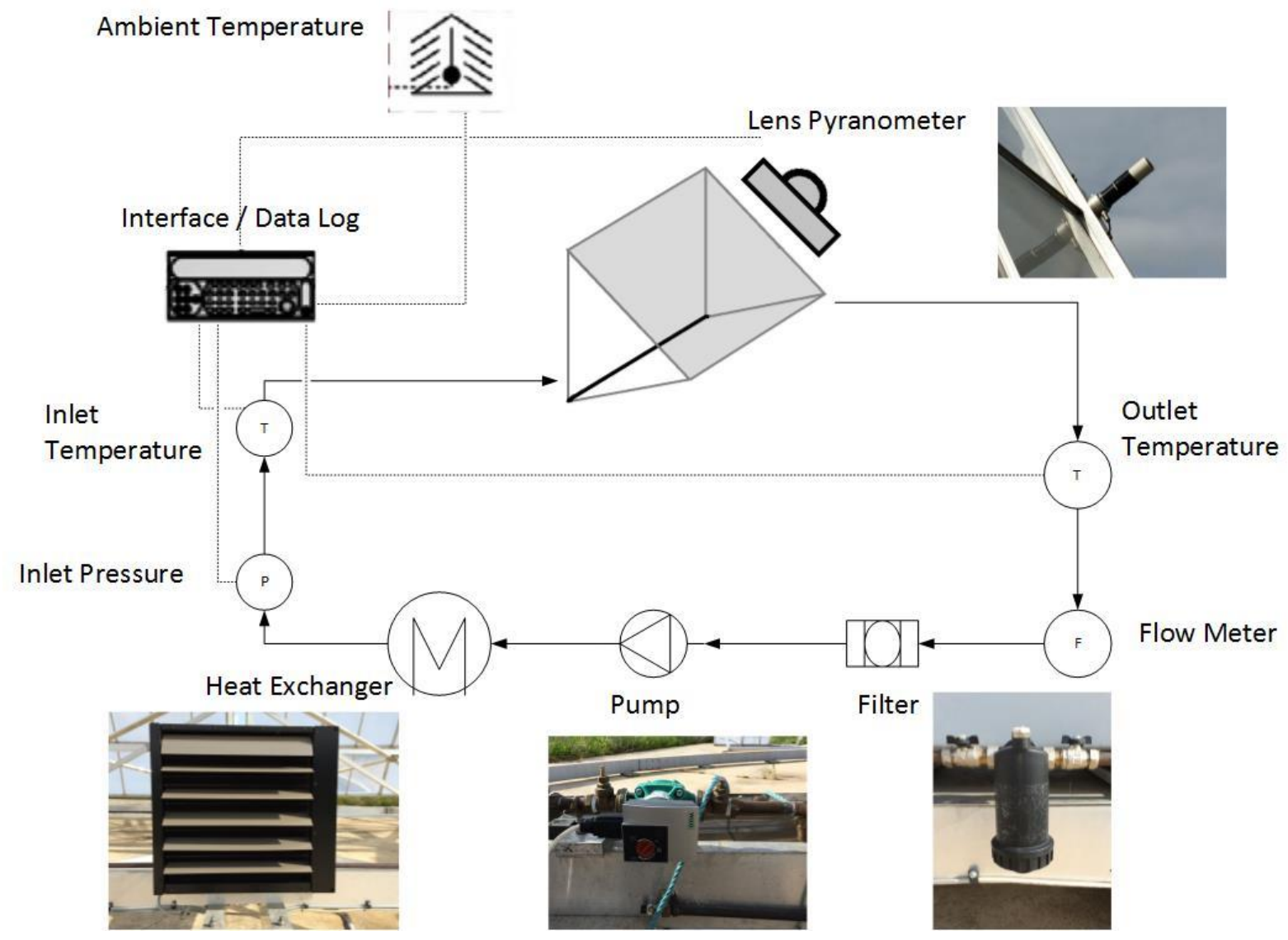

Figure 4: Schematic diagram and measuring system of the dual-axis linear Fresnel lens collector

1. A vortex flow meter. This instrument is used to measure the volumetric flow rate in litres per hours. The accuracy of the device is $0.75 \%$ for a Reynolds number between 4000 and 20000 (turbulent flow). It is supplied with a thermo-resistor device (Pt1000) installed in the vortex sensor. The outlet temperature is measured using this sensor which has a maximum error of $1{ }^{\circ} \mathrm{C}$.

2. A resistance thermometer sensor with an accuracy of $\pm 0.3^{\circ} \mathrm{C}$ is used to measure the fluid temperature at the inlet section of the receiver.

3. A pressure transmitter with an accuracy of $\pm 3.0 \%$ is used to measure the inlet pressure of the fluid.

4. A digital secondary standard pyranometer is installed to measure the solar radiation incident on the lenses surface.

The data is recorded every minute and transmitted to an online platform. The pyranometer installed on the lens frame has been converted into a pyrheliometer to measure the Direct Normal Irradiance (DNI). A collimator tube has been designed and installed in order to eliminate the diffuse radiation and to reduce the circumsolar sky radiation. The collimator has been designed following the procedure presented by Mujahid and Alamoud (1998) who designed a collimator tube for a 
photovoltaic pyrheliometer. They reported that the error in measurements of direct normal radiation can be reduced by $2.3 \%$ using this system. The error band for the pyrheliometer is $\pm 20 \mathrm{~W} / \mathrm{m}^{2}$. The reliability of collimation tubes with respect to the "sun and shade" method (indirect measurement of DNI shading the pyranometer) has been studied by Ijima (2010). The measurements of the converted pyrheliometer have been compared and validated against the data available on the SolarGis platform for the selected location (Solargis, 2010).

\section{Mathematical models}

In order to analyse the performance of the system, a mathematical model was developed and validated. The Fresnel lens system (Lens 1 + Lens 2) was characterized using an optical model implemented in Matlab. The performance of the lenses was evaluated using ray tracing analyses carried out with SolTrace. The heat transfer in the receiver was modelled and simulated using Matlab.

\subsection{Optical model}

The geometry of the Fresnel lenses was analysed using a code implemented in Matlab. This program calculates the inclination of each surface of the prisms with respect to a global reference system. In this case, the origin is considered to be in the middle section of the prototype (see Figure 3).

Assuming that the incident angle ( $i$ ) on the back surface is zero (as in the case of Lens 1 and Lens 2 with negligible tracking error), in each prisms the slope angle $\beta$ is equal to the incident angle on the front surface (See Figure 5).

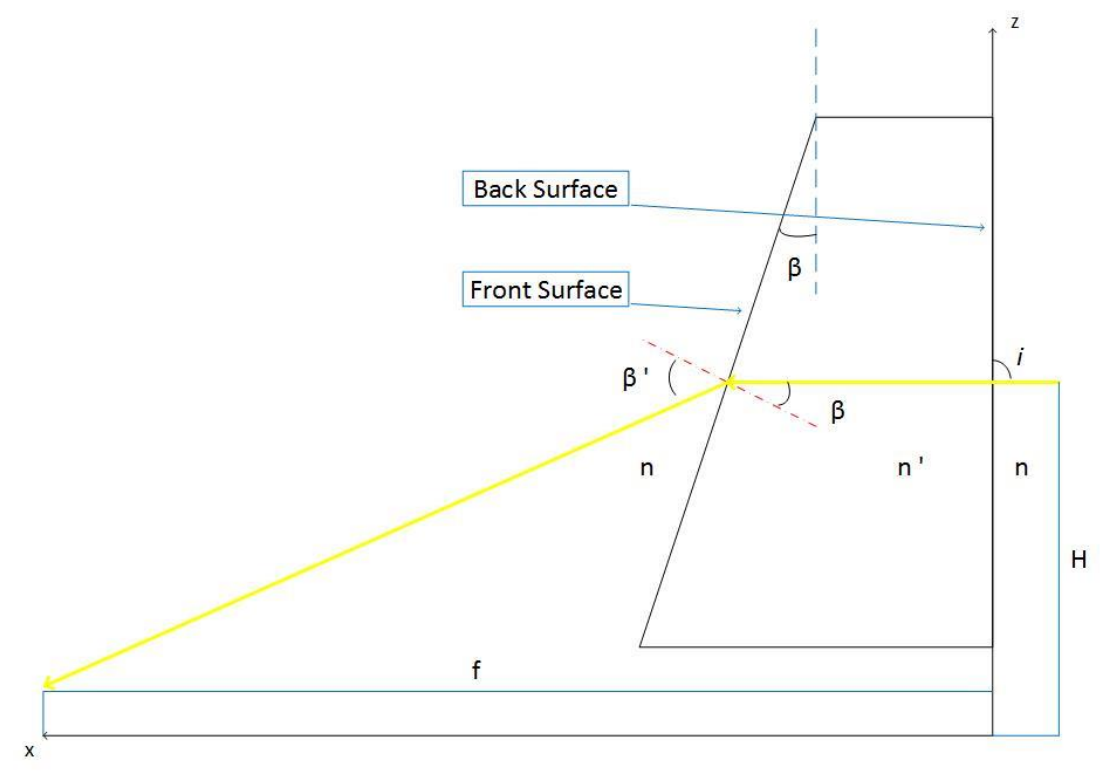

Figure 5: Prims geometry and refraction principle

Furthermore, it can be demonstrated that the refraction angle $\beta$ ' is:

$$
\beta^{\prime}=\tan ^{-1}\left(\frac{H}{f}\right)+\beta
$$

Where $\mathrm{H}$ is the height of the prism with respect to the reference system and $\mathrm{f}$ is the focal length. According to the Snell's law (Leutz and Suzuki, 2001):

$$
\frac{\sin \beta}{\sin \beta^{\prime}}=\frac{n^{\prime}}{n}
$$


The parameters $n$ and $n^{\prime}$ represent the refractive indices and they can be defined for each wavelength in different materials. In this study, $n^{\prime}$ and $n$ are equal to 1 (refractive index of air) and 1.49 for PMMA respectively considering the entire spectrum of light (Leutz and Suzuki, 2001). Therefore, it is possible to calculate the slope angle using the following non-linear equation:

$$
n \cdot \sin \beta-\sin \left(\tan ^{-1}\left(\frac{H}{f}\right)+\beta\right)=0
$$

Finally, the code defines the geometry of the lenses: the position of the prisms is calculated to be used as an input in SolTrace. The ray tracing software also requires the definition of the optical properties of the material. In this study, the PMMA average transmittance for the visible part of the solar spectrum for the lenses is assumed to be 0.92 (Leutz and Suzuki, 2001). The slope error of the prisms have been considered $5 \mathrm{mrad}$ and the Sun Shape has been approximated with a pillbox distribution and the half angle is $5 \mathrm{mrad}$ (Lovegrove and Stein, 2012).

\subsection{Heat transfer model}

The heat transfer model is based on the one dimensional steady state energy balance within the absorber (i.e. the steel pipe) developed by Forristall (2003). Figure 6 shows the thermal resistance network for a cross-section of the absorber.

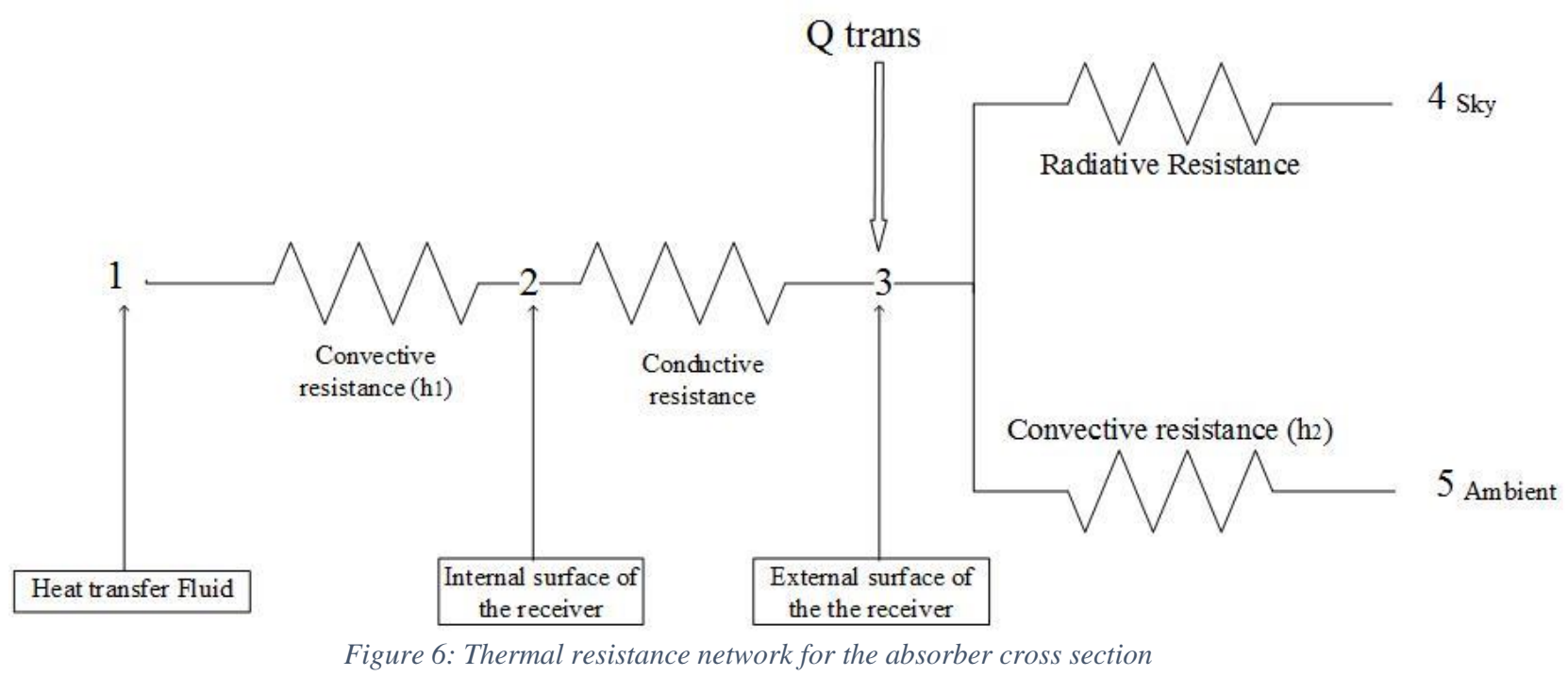

Note that the optical losses have been omitted from the resistance model: Q trans represents the energy transmitted by the lenses. The optical losses in the concentrator are due to absorption and reflection in the plastic material of the lenses. Part of the solar radiation is absorbed by the receiver and transferred to the heat transfer fluid by convection. The rest of the energy is lost to the environment through convection and radiation.

In order to simplify the analysis, the following assumptions are made:

1. Tracking error is negligible.

2. The temperature distribution is uniform around the circumference of the absorber.

3. The variation of the surface temperature along the axial direction of the collector is neglected.

4. The thermal properties of component materials do not vary and those of air and fluid are curve fitted from tables of thermal properties (Holmgren, 2006)

5. The solar absorption is treated as heat flux term. In reality, the solar absorption in the absorber is a volumetric phenomenon.

6. The wind speed was measured close to the collector in Bourne on the corresponding dates. The wind speed data is shown in Figure 7 below. 
7. The pressure of the fluid is constant in the system and estimated as the average of the value measured during the experiments.

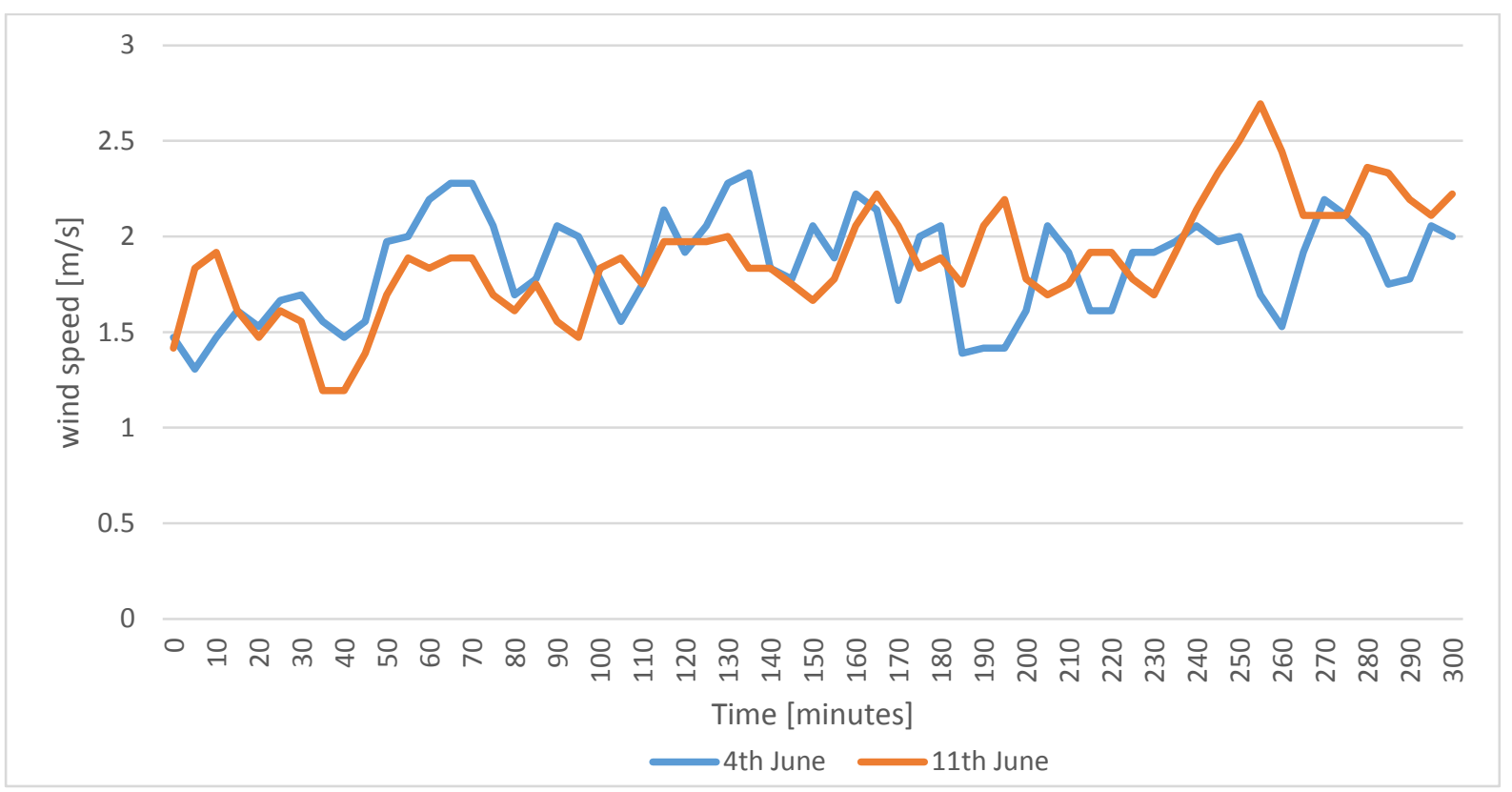

Figure 7: Wind speeds during testing

The energy balance in the receiver pipe is determined by applying the energy conservation principle at each surface of the system (Forristall, 2003). In the equations that follow the numerical subscripts 1-5 refer to locations 1-5 in Figure 6.

$h_{1} \pi D_{2}\left(T_{2}-T_{1}\right)=\frac{2 \pi k_{23}\left(T_{2}-T_{3}\right)}{\ln \left(D_{3} / D_{2}\right)}$

$k_{1}{q^{\prime}}_{\text {trans }}^{\cdot} \eta_{a b s} \alpha_{a b s}=k_{2} h_{2} \pi D_{3}\left(T_{3}-T_{5}\right)+\sigma \varepsilon_{3} \pi D_{3}\left(T_{3}{ }^{4}-T_{4}{ }^{4}\right)+\frac{2 \pi k_{23}\left(T_{2}-T_{3}\right)}{\ln \left({ }_{3} / D_{2}\right)}$

$q_{\text {loss }}^{\prime}=k_{2} h_{2} \pi D_{3}\left(T_{3}-T_{5}\right)+\sigma \varepsilon_{3} \pi D_{3}\left(T_{3}{ }^{4}-T_{4}{ }^{4}\right)$

$\dot{m}\left[c_{\text {p.ave }}\left(T_{\text {out }}-T_{\text {in }}\right)+\frac{1}{2}\left(v_{\text {out }}^{2}-v_{\text {in }}^{2}\right)\right]=\left(q_{\text {trans }}^{\prime} \eta_{\text {abs }} \alpha_{\text {abs }}-q^{\prime} \cdot{ }_{\text {loss }}\right) L$

Where :

- $T_{1}$ is the fluid bulk temperature calculated as the average between the inlet and outlet temperatures

- $T_{2}$ and $T_{3}$ are the internal and external surface temperatures of the receiver

- $T_{4}$ is the equivalent sky temperature approximated as $T_{4}=T_{5}-8$ (Forristall, 2003)

- $T_{5}$ is the ambient temperature.

- $D_{2}$ is the inner diameter of the steel absorber tube

- $D_{3}$ is the outer diameter of the steel absorber tube 
The heat transfer coefficients are defined in Table 1 . The emissivity $\varepsilon_{3}$ of the receiver tube is assumed to be 0.2 (Martinez, 1995). In this model, the absorptance $\alpha_{a b s}$ for carbon steel is assumed equal to 0.38. $\eta_{a b s}$ represents the interception factor determined with the ray tracing analysis.

Table 1: List of heat transfer coefficient used in the model

\begin{tabular}{|c|c|c|c|c|}
\hline Heat Transfer coefficient & Nusselt Number & \multirow{2}{*}{\multicolumn{3}{|c|}{$\begin{array}{l}\text { Kange } \\
\begin{array}{c}2300<R e_{D 2}<4000 \\
0.5<P r_{1}<2000\end{array}\end{array}$}} \\
\hline \multirow[t]{2}{*}{$h_{1}=N u_{D 2} \cdot \frac{k_{1}}{D_{2}}$} & $\begin{aligned} N u_{D 2} & =\frac{f_{2} / 8 \cdot\left(R e_{D_{2}}-1000\right) \cdot P r_{1}}{1+12.7 \cdot \sqrt{f_{2} / 8} \cdot\left(P r_{1}^{2 / 3}-1\right)} \cdot\left(\frac{P r_{1}}{P r_{2}}\right)^{0.11} \\
f_{2} & =\left(1.82 \cdot \log _{10}\left(R e_{D_{2}}\right)-1.64\right)^{-2}\end{aligned}$ & & & \\
\hline & $N u_{D 2}=4.36$ & \multicolumn{3}{|c|}{$R e_{D 2}<2300$} \\
\hline \multicolumn{2}{|c|}{$k_{23}=-0.0309 \cdot T_{23}+60.8989$, where $T_{23}=\frac{T_{2}+T_{3}}{2}$} & \multicolumn{3}{|c|}{ Carbon steel heat conductivity } \\
\hline \multirow{5}{*}{$h_{2}=N u_{D 3} \cdot \frac{k_{35}}{D_{3}}$} & \multirow{5}{*}{$\begin{array}{l}N u_{D 3}=C \cdot R e_{D 3}^{m} \cdot P r_{5}{ }^{n} \cdot\left(\frac{P r_{5}}{P r_{3}}\right)^{0.25} \\
0.7<P r_{5}<500, \text { and } 1<R e_{D 3}^{<}<10^{6}\end{array}$} & $R e_{D 3}$ & C & $m$ \\
\hline & & $1-40$ & 0.75 & 0.4 \\
\hline & & $40-1000$ & 0.51 & 0.5 \\
\hline & & $\frac{1000-200000}{200000-1000000}$ & 0.26 & 0.6 \\
\hline & & \multicolumn{3}{|c|}{$\begin{array}{l}n=0.37, \text { for } \operatorname{Pr} \leq 10 \\
n=0.36, \text { for } \operatorname{Pr}>10\end{array}$} \\
\hline
\end{tabular}

Note that $f_{2}$ is the friction coefficient at the inner surface of the pipe. $P r_{1}$ is the Prandtl number at $T_{1}$ and $P_{2}$ is the Prandtl number at $T_{2} . R e_{D 3}$ is the Reynolds number of the air calculated at the absorber pipe external diameter. $k_{35}$ is the thermal conductivity of air at $T_{35}$, where $T_{35}=\left(T_{3}+T_{5}\right) / 2$.

The irradiance incident on the lenses has been reduced by $10 \%$ (the parameter $k_{1}$ is equals to 0.9 ). The reduction takes into consideration the losses due to manufacturing error in the prisms (Davis and Kuhnlenz, 2007). Furthermore, the optical characteristic defined in the model refer to a fixed wavelength of the light. In reality, the solar spectrum is larger and lenses cannot be optimized for each wavelength. Similarly, the model uses the theoretical value of the transmittance for the PMMA, but the material behaves differently in real conditions. The shadowing effect due to the frame are also considered at this stage.

The parameter $k_{2}$ has been assumed to be 0.95 . The front pipe is heated up more than the back surface and therefore the cooling effect of the convection on the pipe is reduced with respect to the model assumptions.

Given the fluid inlet temperature at the receiver at each time step, equations (1) - (4) form a set of nonlinear algebraic equations to solve the four unknowns of the model: $T_{\text {out }}, T_{2}, T_{3}, q_{\text {heatloss. }}^{\prime}$ The model implemented in this study considers also the thermal losses occurring in the recirculation system. An empirical equation has been developed to calculate the temperature of the fluid at the inlet section of the receiver pipe:

$$
T_{\text {in }}(t+1)=0.6 \cdot T_{\text {in }}(t)+0.4 \cdot T_{\text {out }}(t)-1
$$

Equation (8) approximates the effect mixing of the heated fluid in the tank and the temperature drops in the recirculation system as previously described. The equation has been selected to be linear rather than logarithmic in order to simplify the model. The thermal inertia of our system including the tank does not require a more complex equation at this stage. However part of our further work will be to focus on that aspect, which will become relevant as we scale up and increase the volume of liquid used in the thermal loop. The values of the parameters have been determined by fitting experimental data. 


\section{Models Validation and Performance curve}

The prototype was tested in June 2015 in Bourne in the UK (Latitude $52^{\circ} 46^{\prime} 20.8056^{\prime \prime} \mathrm{N}$ ). The data gathered during the testing campaign was used for the validation of the thermal model. The experimental measures were compared to the results of the thermal model. The model has been used to define the performance curve and evaluate the thermal losses in the system. A specific experiment has also been conducted to understand the performance of the lens array. The results of the tests have been compared to the ray tracing simulations to validate the optical model.

\subsection{Validation of the Optical model}

The optical model was validated using experimental data. Since the design is symmetric and to simplify the experiment procedure, only a small section of the frame was considered (Figure 8).

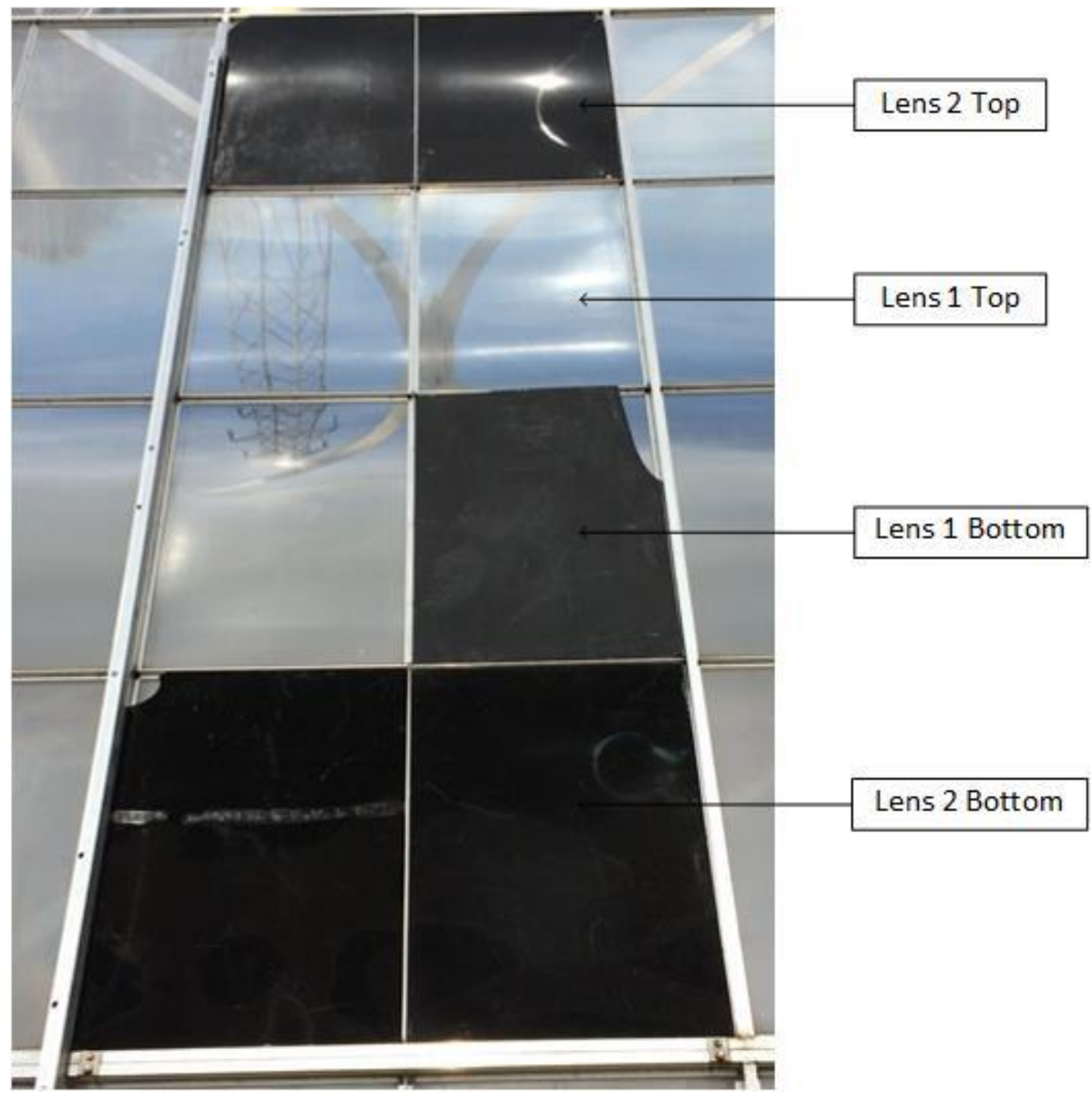

Figure 8: Prototype set-up for experiment (21st April 2016)

In this case, the optical system consisted of three Lens 1 components. For experimental purpose, one of the Lens 1 has been shadowed. A flat panel was installed at 2.5 metres from the lenses on the optical axis plane which coincide with the receiver tube axis. This setup was implemented in SolTrace using the optical model previously developed. Figure 9 reports the measurement of the concentrated 
light beam and the flux distribution on the panel calculated with the ray tracing analysis. It should be noted that the flux distribution was not measured experimentally. Rather, for validation purposes, the beam width was compared to the width of the beam generated using the ray - tracing software.

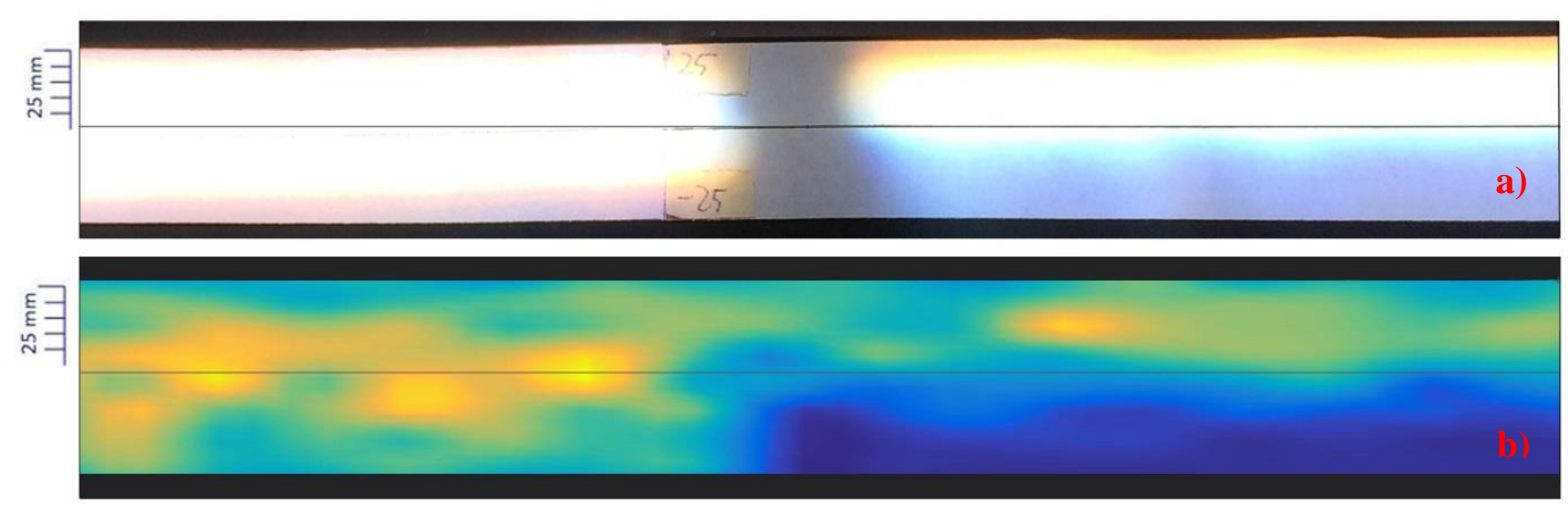

Figure 9: a) Concentrated beam during experiment (21st April 2016) b) Flux distribution according to ray tracing simulation

The two Lens 1 concentrated the normal incident light into a $50 \mathrm{~mm}$ wide beam, symmetrical with respect to the horizontal axis of the target (see Figure 9a on the left). The single Lens 1 top component is able to refract a $25 \mathrm{~mm}$ concentrated beam above the horizontal axis (see Figure 9a on the right). The light beam simulated with the ray tracing analysis is reported in Figure 9b. It can be seen that the model predicts the behaviour of the lenses.

Note that there is a gap between the two beams. This interval is caused by the shadowing effect of the metal frame between two lenses (see Figure 8). The distance between the lenses has been simulated in SolTrace. As a result, the model predicts a less intense flux between the beams (Figure $9 b$ ).

The SolTrace model has been used to calculate the percentage of solar radiation incident on the lenses which reaches the receiver pipe. The parameter is named interception factor $\left(\eta_{a b s}\right)$ (Forristall, 2003). The interception factor for the system analysed in this study is $53 \%$.

The shadowing effect has also been analysed using the model. Referring to the experimental set-up (Figure 8), simulations without gap in the lenses give an interception factor of $40.43 \%$. When the frame is considered, the calculated interception factor is $39.33 \%$. The shadowing effect contribute to a reduction of less than $1 \%$ in the light refracted onto the target. The shadowing effect have been considered in the thermal model.

\subsection{Validation of the Thermal model}

The system has been tested in June 2015. Two days have been considered for the validation of the thermal model when the solar radiation was more constant during the testing period. The current thermal model has been used to simulate the heating phase of the system. Figure 10 reports the comparison with the measured and calculated values for the $4^{\text {th }}$ of June. This day the system was tested for 116 minutes with an average DNI of $898.5 \mathrm{~W} / \mathrm{m}^{2}$. The model was tested also on the $11^{\text {th }}$ of June for 320 minutes when the average direct normal irradiance was $793 \mathrm{~W} / \mathrm{m}^{2}$. The results are reported in Figure 11. 


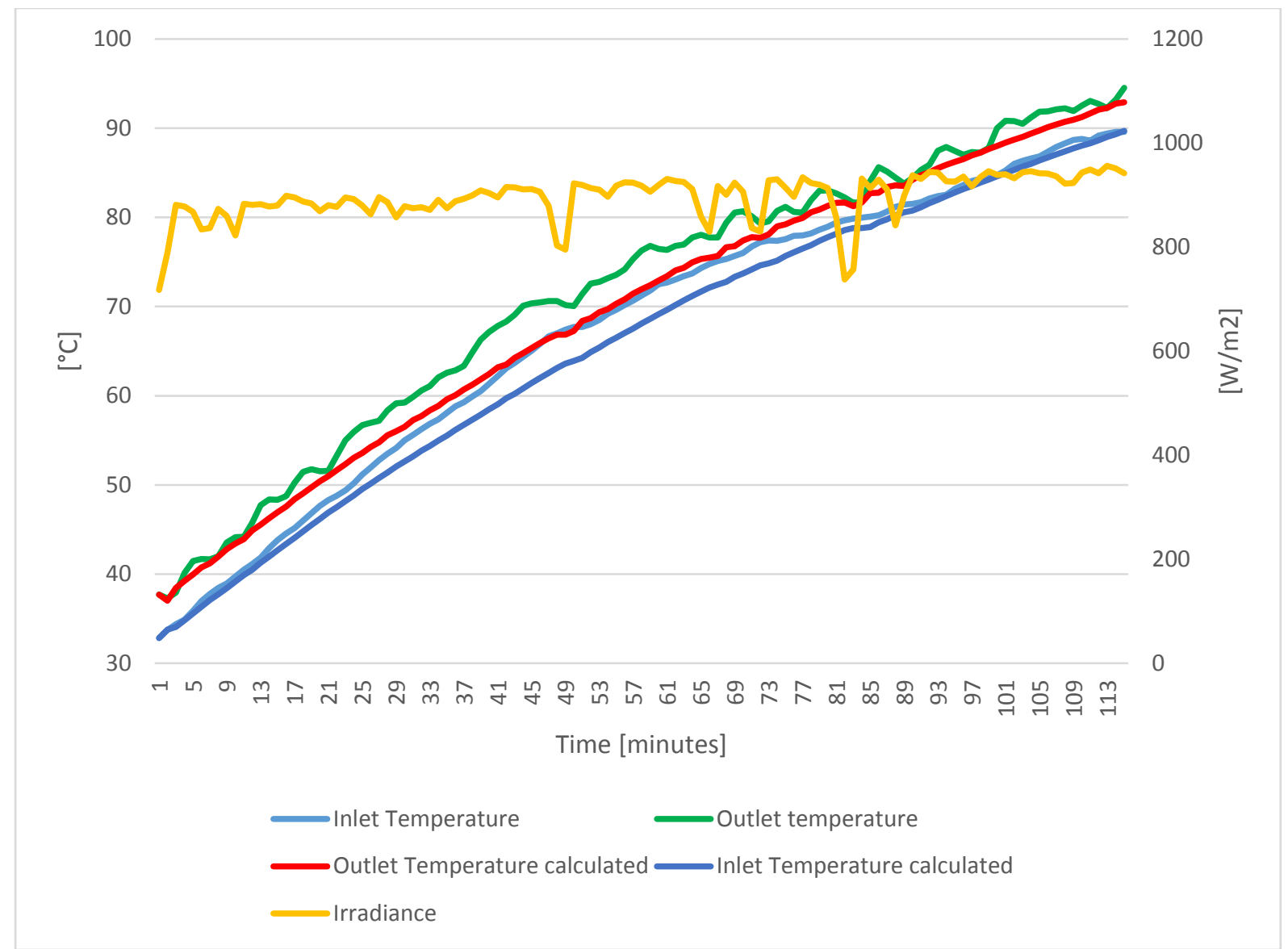

Figure 10: Measured and calculated temperature profiles for the $4^{\text {th }}$ of June 2015

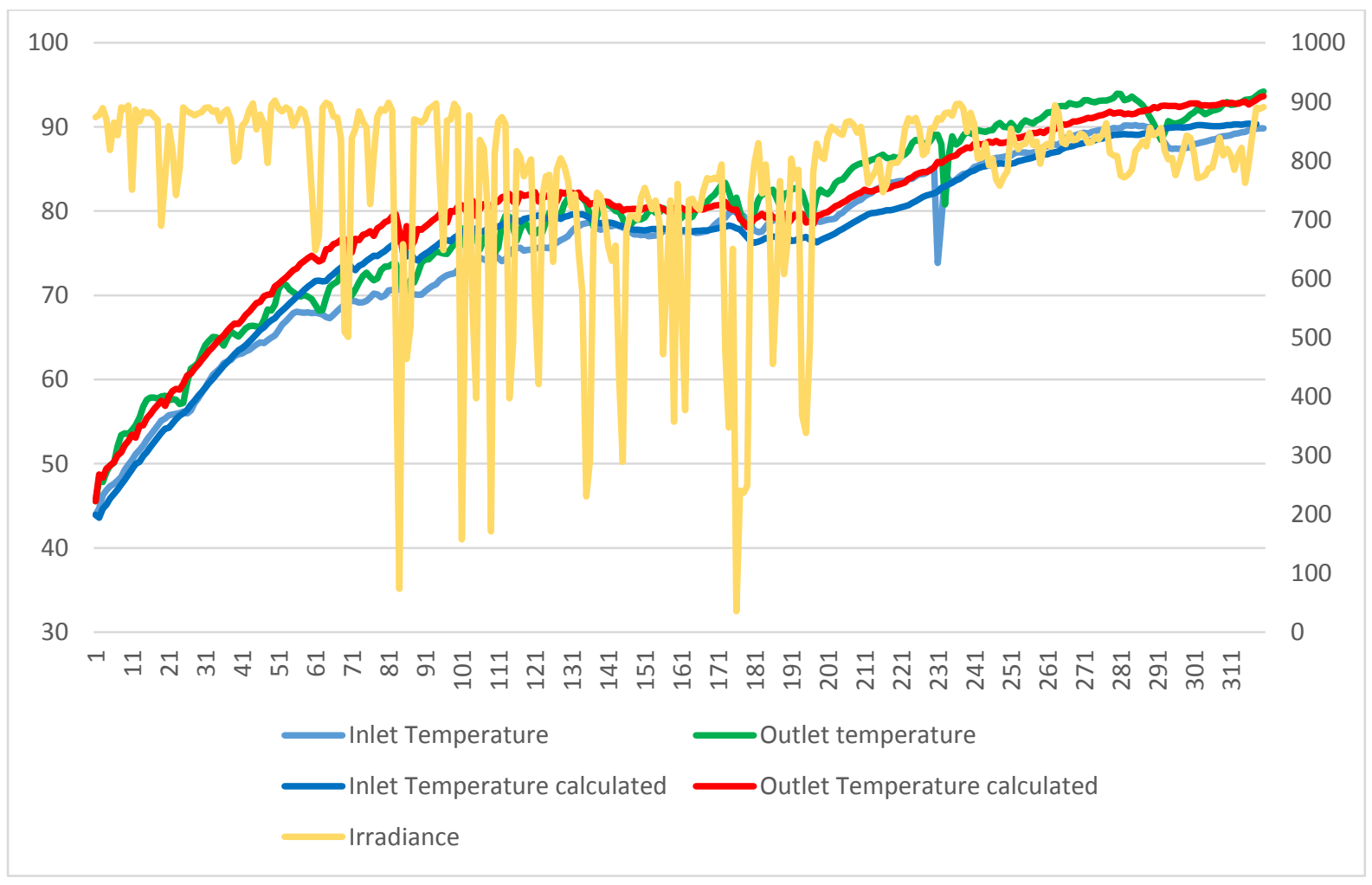

Figure 11: Measured and calculated temperature profiles for the $11^{\text {th }}$ of June 2015 
The absolute error is defined as the absolute value of the difference between the measured and the calculated value. The relative absolute error is defined as the absolute error as a percentage of the measured value. On the $4^{\text {th }}$ June 2015 , the average absolute error in outlet temperature calculated by the model is $2.13{ }^{\circ} \mathrm{C}$ and the relative absolute error is $3 \%$. For the $11^{\text {th }}$ of June the average absolute error in outlet temperature is $2.50{ }^{\circ} \mathrm{C}$ and the relative absolute error is $2.9 \%$.

The model is oversensitive to the variations in the irradiance, compared to the response of the real system. This is related to the assumption of the model of a steady state condition.

Figure 12 represents the analysis of the error with respect to the irradiance for the $11^{\text {th }}$ of June.

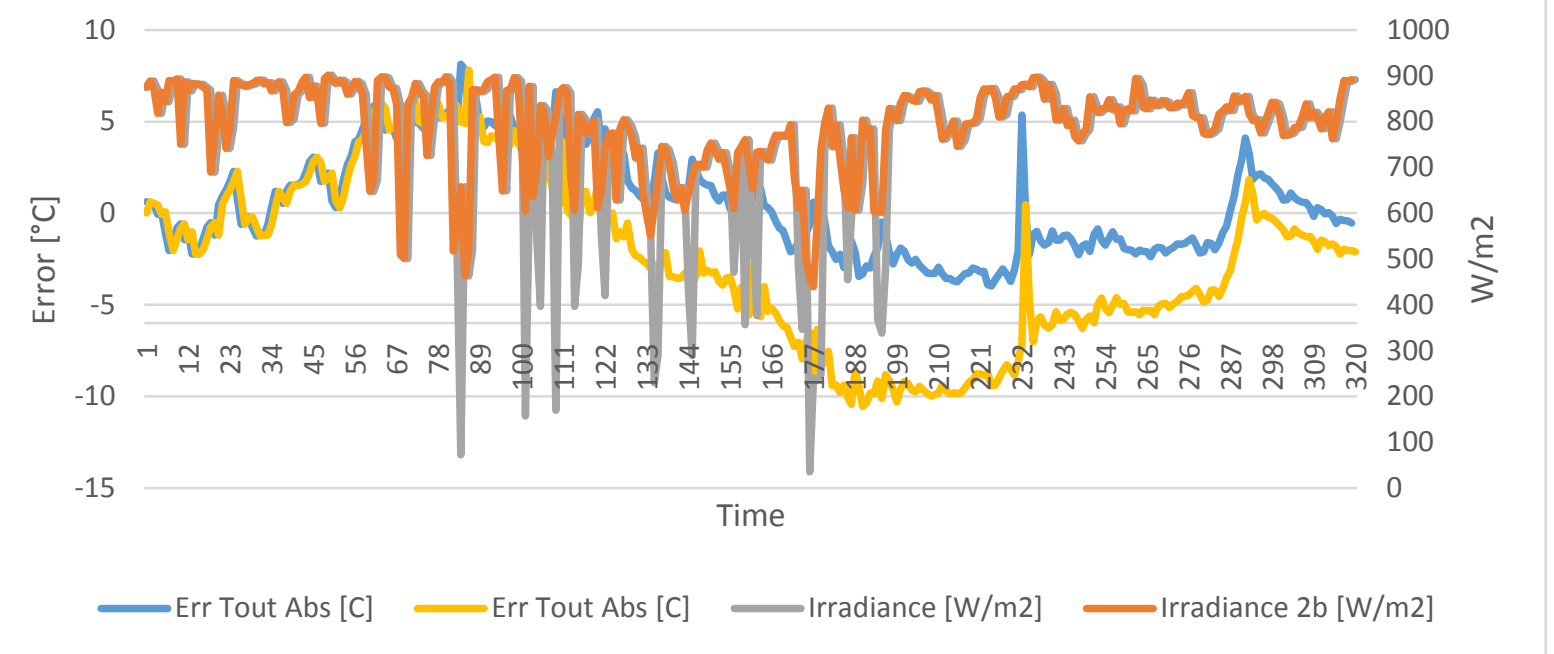

Figure 12: Comparison of experimental and simulation results for the 11th of June 2015

The model underestimates the losses when the irradiance is constant and overestimates the value of the outlet temperature when the irradiance is more variable. In particular, the model is inaccurate for radiation level below $400 \mathrm{~W} / \mathrm{m}^{2}$. Therefore the curve of the irradiance for the $11^{\text {th }}$ of June has been approximated to reduce the effect of the variability. In practical terms this means that the model does not cope well when clouds obscure the sun, since it does not take into account the thermal inertia of the system. As a result the curve of the irradiance for the 11th of June has been approximated (smoothed) to eliminate sharp reductions in solar irradiance. This is shown in Figure 13, where the smoothed irradiance plot for the time period up to 141 minutes is shown as "Irradiance $2 \mathrm{~b}$ ". Both the original Irradiance and Irradiance $2 \mathrm{~b}$ are also plotted for the total duration in Figure 12.

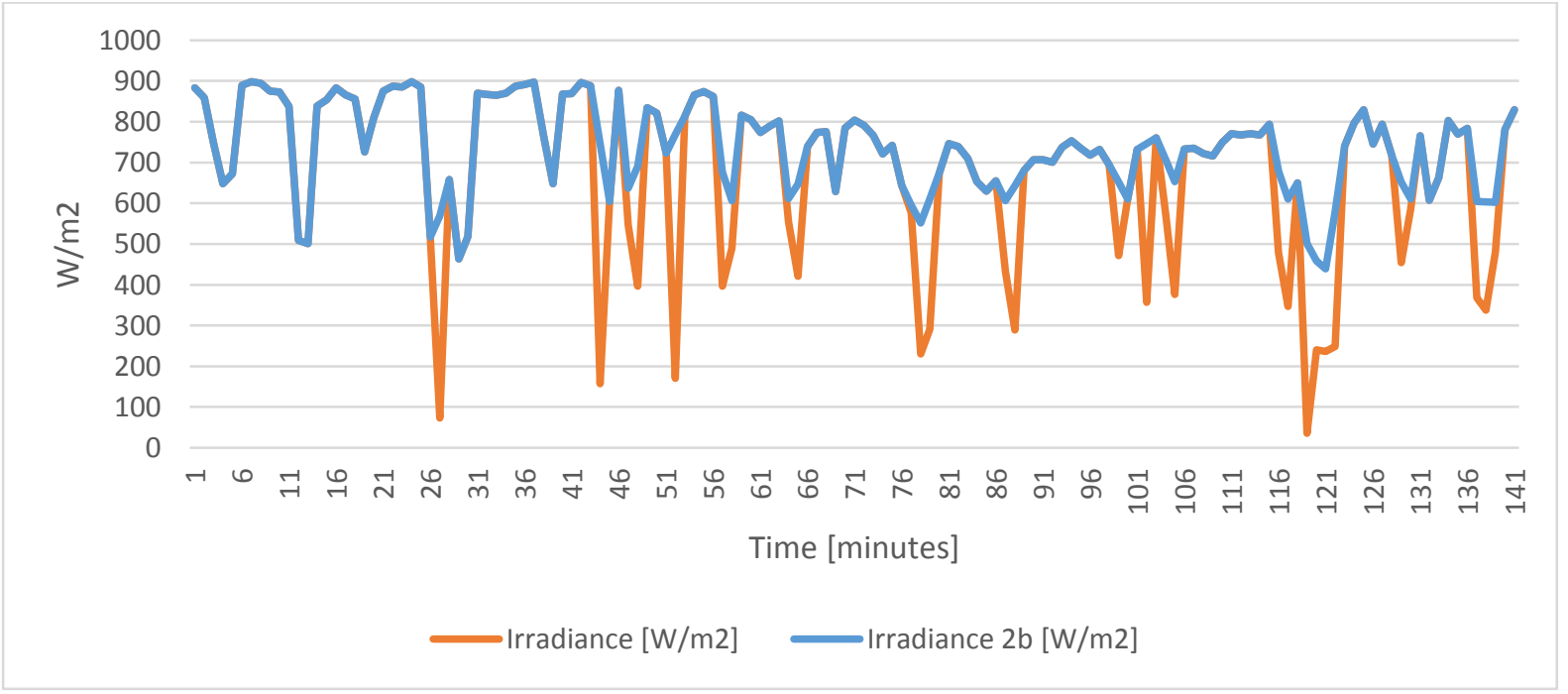

Figure 13: Irradiance approximation 
Future work will focus on the optimization of the model. Further testing will be needed to understand the losses the receiver pipe and in the recirculation system.

\subsection{Performance Curve and losses analysis}

The instantaneous thermal efficiency can be estimated as follow (Zhai et al., 2010):

$$
\eta_{\text {ist }}=\frac{\dot{m} \cdot c_{p, \text { ave }} \cdot\left(T_{\text {out }, \text { calc }}-T_{\text {in }}\right)}{A_{\text {lens }} \cdot G_{\text {DNI }}}
$$

$T_{\text {out,calc }}$ is the calculated temperature at the outlet section of the receiver pipe. Six inlet temperature levels were considered: $40^{\circ} \mathrm{C}, 50^{\circ} \mathrm{C}, 60^{\circ} \mathrm{C}, 70^{\circ} \mathrm{C}, 80^{\circ} \mathrm{C}$, and $90^{\circ} \mathrm{C}$. The calculated global efficiency was plotted against the parameter defined as (Zhai et al., 2010):

$$
x=\frac{\left(\left(T_{\text {in }}+T_{\text {out }}\right) / 2-T_{\text {amb }}\right)}{G_{D N I}}
$$

Figure 14 shows the performance curve of the current prototype.

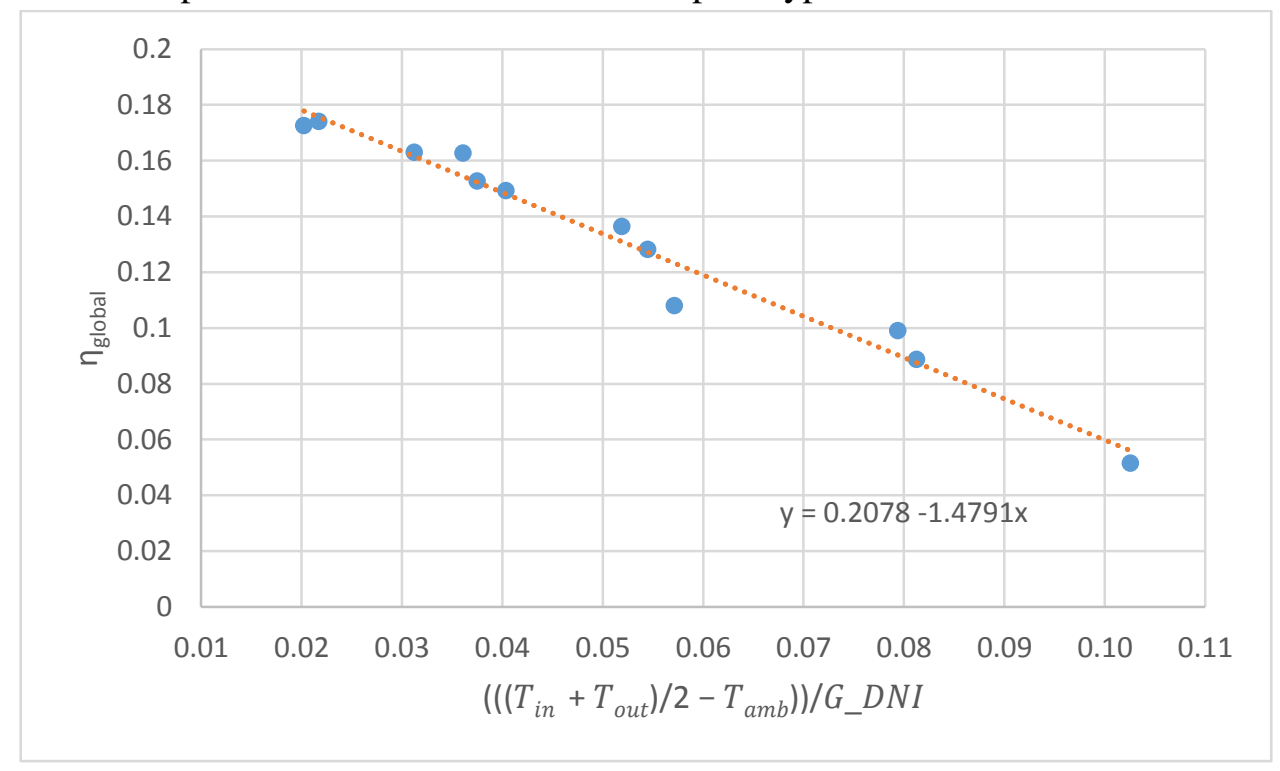

Figure 14: Performance Curve

The uncertainty of the global efficiency of the system can be calculated using the following equation (Zhai et al, 2010).

$$
\frac{d \eta_{\text {ins }}}{\eta_{\text {ins }}}=\frac{d \dot{m}}{\dot{m}}+\frac{d T_{0}}{T_{0}-T_{i}}-\frac{d T_{i}}{T_{0}-T_{i}}-\frac{d I_{b}}{I_{b}^{2}}
$$

Substituting the measured data and the error in the measurement instrument from section 2.1.2, we determine the maximum relative error to be $16.0 \%$.

Previous prototype based on single axes tracking showed better performance at similar temperature levels (Zhai et al., 2010). Therefore, a detailed analysis of the losses in the system was carried out for the reported days. Figure 15 reports the energy losses breakdown for the $4^{\text {th }}$ June. 


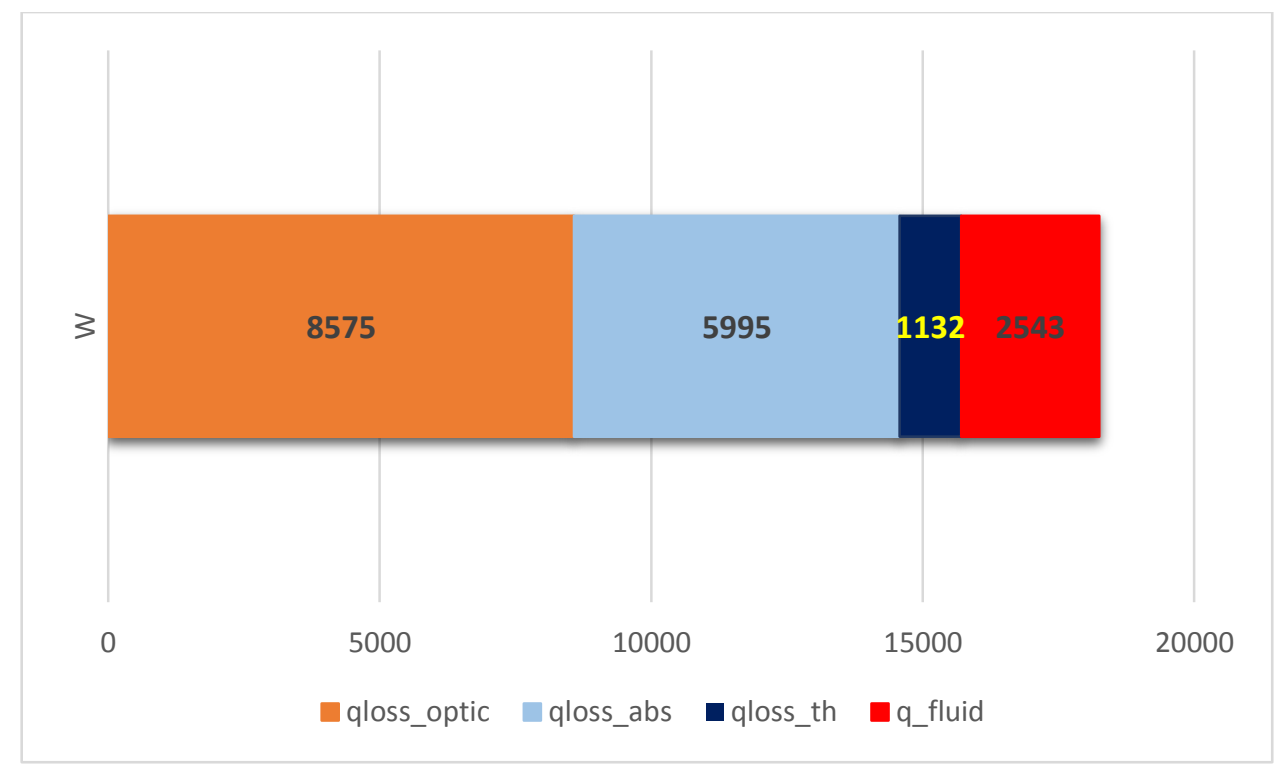

Figure 15: Thermal losses breakdown for the experiment on the 4th June 2015

The optical losses (qloss_optic) represent the energy lost due to absorption, reflection and diffraction in the lenses. The effect of the light spectrum and the material properties are also affecting the refraction process. The heat which is not absorbed by the pipe is considered in the absorption losses (qloss_abs). The thermal losses (qloss_th) represent the sum of the convective and radiative losses from the absorber pipe (see Equation (6)). The thermal power actually transmitted to the fluid is reported as "q_fluid". The losses in the lenses accounts for the $47 \%$ of the total energy lost in the collector (see Figure 16).

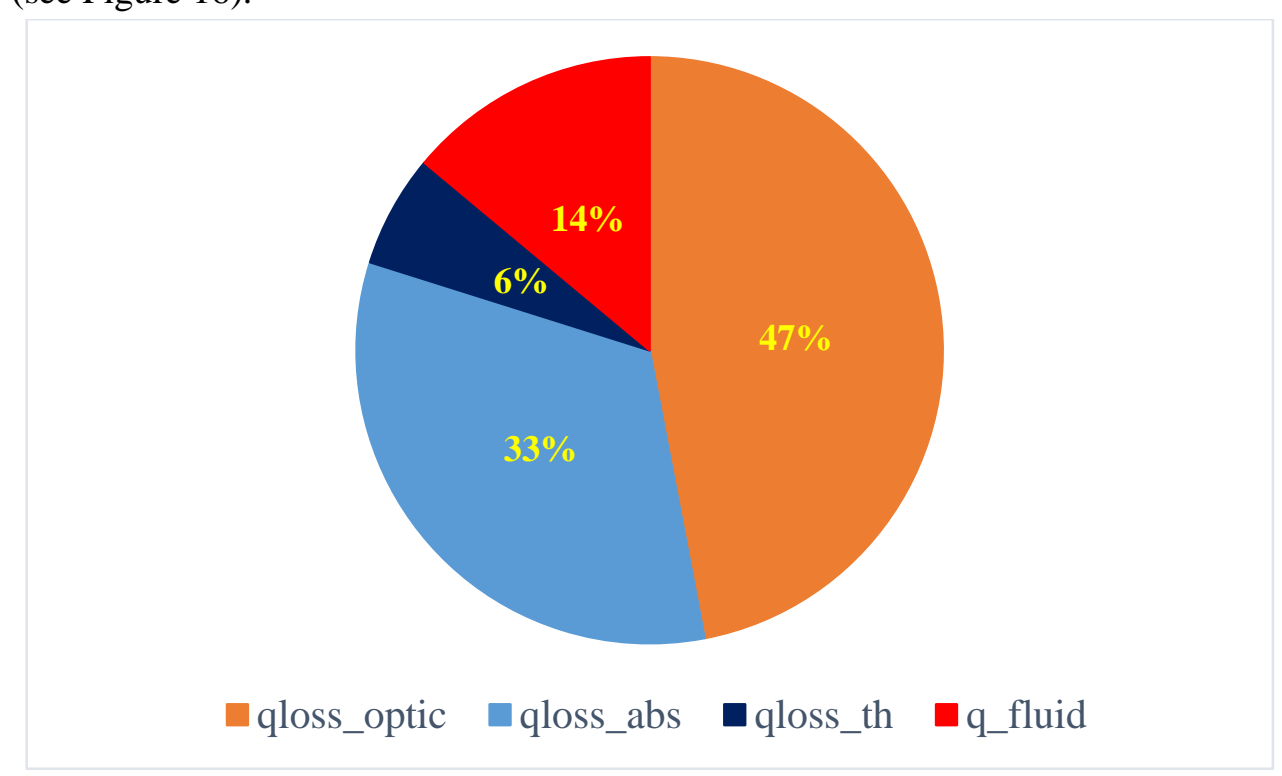

Figure 16: Thermal losses analysis for the experiment on the 4th June 2015

The optical efficiency has been calculated to be about $53 \%$ in design condition for the current set of lenses. Note that the effect of dust on the lenses has not been considered in this work. Future study will be carried out to analyse specifically the impact of uncleaned PMMA surface on the optical efficiency of the system. One third of the total losses (33\%) is due to the low absorption in the carbon steel pipe absorber. The receiver installed on the prototype is a schedule 40 pipe without selective coating to improve the absorption of the solar radiation and no glass envelope to reduce convective losses. The heat transferred to the ambient through convection and radiation from the pipe is lower with respect to other loss mechanisms (only 6\%). 


\section{Conclusions and future works}

In this study, the performance of a PMMA acrylic Fresnel lens solar collector, based on a dual-axis tracking system, has been analysed. A mathematical model, including the optical model of the concentrator and the heat transfer model of the receiver pipe and recirculation system was developed and validated using experimental data. The performance curve of the collector has been derived for temperatures between $40^{\circ} \mathrm{C}$ and $90^{\circ} \mathrm{C}$.

The calculated global efficiency of the collector is limited to less than $20 \%$. The main sources of energy losses are due to: the optical losses in the lens system (47\%) and the low solar absorptance of the absorber pipe (33\%). The thermal losses due to radiation and convection, account for only $6 \%$ as relatively low temperatures (up to $90^{\circ} \mathrm{C}$ ) are reached on the absorber pipe to promote radiation and convection heat transfer.

While this technology is promising as a low cost concentrated solar collector for medium temperatures, the results of this study highlighted that it can be improved by reducing the absorption losses in the receiver pipe. It is recommended to install a solar selective coated evacuated receiver to reduce the convective losses from the receiver pipe and increase the solar absorption. Previous works showed that single tracking linear Fresnel lenses collector with evacuated tube receiver achieved a global efficiency up to 55\% (Zhai et al., 2010). Furthermore, the use of insulation will reduce the thermal losses in the recirculation system. It should be noted that the performance of the experimental prototype is below expectations partly due to its location and the non-optimal optical design of the lenses. Linear Fresnel lenses have been proven to achieve higher temperatures, as presented most recently by Linaric (2013). The target temperature for the next prototype is above $200^{\circ} \mathrm{C}$ to match the requirements for industrial heat processes $(57 \%$ of industrial heat demand requires temperatures below $400^{\circ} \mathrm{C}$ (Rawlins and Ashcroft, 2013)).

Both single and dual axis tracking remain an option. The current use of dual-axis tracking is an additional cost. However, simple and low cost closed loop dual-axis tracking systems can be implemented with open source software and hardware. Furthermore, additional modules of lenses can be clustered (as in the current prototype) to be moved using the existing tracking and control system. An improved version of the prototype is currently be designed and further tests will be conducted. The goal is to obtain a commercially attractive product based on the acrylic Fresnel lens principle for applications in industrial process heat processes, with a target cost of $€ 300 / \mathrm{m} 2$ or $0.04-0.07 € / \mathrm{kWh}$ (Ivancic et al, 2014).

\section{Acknowledgment}

This work has been realised thank to the Knowledge Transfer Partnership programme sponsored by Innovate UK and Lark Energy (Partnership ID 9630).

\section{References:}

Al-Jumaily K. E. J., Al-Kaysi, M. K.A., 1998. The study of the performance and efficiency of flat linear Fresnel lens collector with sun tracking system in Iraq. Renewable Energy.14, 41-8.

Boyd, DA, Gajewski, R, Swift, R, 1976. A cylindrical blackbody solar energy receiver. Solar Energy. $18,395-401$.

Davis, A, Kuhnlenz, F, 2007. Optical Design using Fresnel Lenses: basic principles and some practical examples. Optik \& Photonik. 2(4), 52-55. 
Fernández-García, A., Zarza, E., Valenzuela, L. and Pérez, M, 2010. Parabolic-trough solar collectors and their applications. Renewable and Sustainable Energy Reviews. 14, 1695-1721.

Forristall, R., 2003. Heat Transfer Analysis and Modelling of a Parabolic Trough Solar Receiver Implemented in Engineering Equation Solver. NREL Technical Report.

Franc F, Jirka V, Maly’ M, Náblek, B., 1986. Concentrating collectors with flat linear Fresnel lenses. Solar \& Wind Technology. 3(2), 77-84.

Holmgren, M., 2006. Xsteam for Matlab. Available online: www.X-eng.com. Accessed: June 2015.

Ijima O, 2010. Calibration of pyranometer by Collimation tube method. Radiation Section, Atmospheric Environment Division, Global Environment and Marine Department, Japan Meteorological Agency (JMA), IPC-XI, Davos, Switzerland

IRENA, 2015. "Solar Heat for Industrial Processes: Technology Brief". Available online: http://www.irena.org/DocumentDownloads/Publications/IRENA_ETSAP_Tech_Brief_E21_Solar_He at_Industrial_2015.pdf. Accessed June 2016.

Ivancic A., Mugnier D., Werner Weiss G.S., 2014. Solar Heating and Cooling Technology Roadmap. Available online (www.rhc-platform.org)

Kumar, V, Shrivastava, R.L., Untawale, S.P., 2015. Fresnel lens: A promising alternative of reflectors in concentrated solar power. Renewable and Sustainable Energy Reviews. 44, 376-390.

Leutz, R., Suzuki, A., 2001. Nonimaging Fresnel lenses: design and performance of solar concentrators. Springer, Berlin, New York.

Lin, M., Sumathy, K., Dai, Y.J., Zhao, X.K, 2014. Performance investigation on a linear Fresnel lens solar collector using cavity receiver. Solar Energy. 107, 50-62.

Linaric, D., 2013. EFISOL Report Summary. Available online:

http://cordis.europa.eu/result/rcn/58185_en.html . Accessed June 2016.

Lovegrove, K., Stein, W., 2012 Concentrating Solar Power Technology Principles, Developments and Applications. Woodhead Publishing Series in Energy.

Manikumar, R., Valan Arasu, A., Jayaraj, S., 2014. Numerical simulation of a trapezoidal cavity absorber in the linear Fresnel reflector solar concentrator system. International Journal of Green Energy. 11(4), 344-363.

Martinez, I., 1995. Available online: webserver.dmt.upm.es/ isidoro/dat1/Thermooptical.pdf . Accessed June 2016.

Mujahid, A. M., Alamoud, A. R. M., 1988. An easily designed and constructed photovoltaic pyrheliometer. Solar \& Wind Technology. 5(2), 127-130. 
Rawlins, J., Ashcroft, M., 2013. "Small-scale Concentrated Solar Power: A review of current activity and potential to accelerate deployment". Carbon Trust Available online: https://www.gov.uk/government/uploads/system/uploads/attachment_data/file/191058/small_scale_co ncentrated solar_power_carbon trust.pdf. Accessed June 2016

Reda, I., Afshin, A., 2008. Solar Position Algorithm for Solar Radiation Applications. NREL Technical Report.

Solargis s.r.o., 2010. Online data and tools for solar energy projects. Available online: http://solargis.info/ Accessed June 2015.

Taibi, E., Gielen, D., Bazilian, M. B., 2010, "Renewable Energy in Industrial Applications: An assessment of the 2050 potential". United Nations Industrial Development Organization. Available online:

http://www.unido.org/fileadmin/user_media/Services/Energy_and_Climate_Change/Energy_Efficienc y/Renewables \%20Industrial \%20Applications.pdf . Accessed June 2016

Xie, W.T., Dai, Y.J., Wang R.Z., Sumathy, K, 2011. Concentrated solar energy applications using Fresnel lenses: A review. Renewable and Sustainable Energy Reviews. 15, 2588- 2606.

Zhai, H., Dai, Y.J., Wu, J.Y., Wang, R.Z., Zhang L.Y, 2010. Experimental investigation and analysis on a concentrating solar collector using linear Fresnel lens. Energy Conversion and Management. 51, $48-55$.

Zheng, H., Feng, C, Su, Y, Dai, J., Ma, X, 2010. Design and experimental analysis of a cylindrical compound Fresnel solar concentrator. Solar Energy. 107, 26-37 


\section{Theoretical and experimental analysis of} an innovative dual-axis tracking linear Fresnel lenses concentrated solar

\section{thermal collector}

\section{Perini, Simoni}

\section{Elsevier}

Simoni Perini, Xavier Tonnellier, Peter King, Christopher Sansom, Theoretical and experimental analysis of an innovative dual-axis tracking linear Fresnel lenses concentrated solar thermal collector, Solar Energy, Volume 153, 1 September 2017, Pages 679-690

http://dx.doi.org/10.1016/j.solener.2017.06.010

Downloaded from Cranfield Library Services E-Repository 